\title{
Cosmesis and body image after single-port laparoscopic or conventional laparoscopic cholecystectomy: a multicenter double blinded randomised controlled trial (SPOCC-trial)
}

Daniel C Steinemann ${ }^{1 *}$, Dimitri A Raptis ${ }^{1}$, Georg Lurje ${ }^{1}$, Christian E Oberkofler ${ }^{1}$, Roland Wyss ${ }^{2}$, Adrian Zehnder ${ }^{2}$, Mickael Lesurtel ${ }^{1}$, René Vonlanthen ${ }^{1}$, Pierre-Alain Clavien ${ }^{1}$ and Stefan Breitenstein ${ }^{1 *}$

\begin{abstract}
Background: Emerging attempts have been made to reduce operative trauma and improve cosmetic results of laparoscopic cholecystectomy. There is a trend towards minimizing the number of incisions such as natural transluminal endoscopic surgery (NOTES) and single-port laparoscopic cholecystectomy (SPLC). Many retrospective case series propose excellent cosmesis and reduced pain in SPLC. As the latter has been confirmed in a randomized controlled trial, patient's satisfaction on cosmesis is still controversially debated.

Methods/Design: The SPOCC trial is a prospective, multi-center, double blinded, randomized controlled study comparing SPLC with 4-port conventional laparoscopic cholecystectomy (4PLC) in elective surgery. The hypothesis and primary objective is that patients undergoing SPLC will have a better outcome in cosmesis and body image 12 weeks after surgery. This primary endpoint is assessed using a validated 8-item multiple choice type questionnaire on cosmesis and body image. The secondary endpoint has three entities: the quality of life 12 weeks after surgery assessed by the validated Short-Form-36 Health Survey questionnaire, postoperative pain assessed by a visual analogue scale and the use of analgesics. Operative time, surgeon's experience with SPLC and 4PLC, use of additional ports, conversion to 4PLC or open cholecystectomy, length of stay, costs, time of work as well as intraand postoperative complications are further aspects of the secondary endpoint. Patients are randomly assigned either to SPLC or to 4PLC. Patients as well as treating physicians, nurses and assessors are blinded until the 7th postoperative day. Sample size calculation performed by estimating a difference of cosmesis of 20\% (alpha $=0.05$ and beta $=0.90$, drop out rate of $10 \%$ ) resulted in a number of 55 randomized patients per arm.
\end{abstract}

Discussion: The SPOCC-trial is a prospective, multi-center, double-blind, randomized controlled study to assess cosmesis and body image after SPLC.

Trial registration: (clinicaltrial.gov): NCT 01278472

\section{Background}

Since the first laparoscopic cholecystectomy in 1985, the laparoscopic approach has emerged as the treatment of choice for symptomatic gallstone disease [1,2]. Advantages of laparoscopic compared with open surgery include improved cosmesis, pain control, shorter convalescence

\footnotetext{
* Correspondence: daniel.steinemann@gmx.ch; stefan.breitenstein@usz.ch 'Department of Surgery, Division of Visceral and Transplantation Surgery, University Hospital Zurich, 8091 Zurich, Switzerland

Full list of author information is available at the end of the article
}

and the absence of the formation of intra-abdominal adhesions.

Traditionally laparoscopic cholecystectomy is performed with four ports. In an attempt to further reduce operative trauma and improve cosmetic results following laparoscopic cholecystectomy, there has been a trend toward minimizing the number of incisions and ports required. These new operative techniques are called natural orifice transluminal endoscopic surgery (NOTES) $[3,4]$ or singleport laparoscopic surgery (SPLC) [5] and were developed

\section{Ciomed Central}


in order to improve cosmesis and further reduce the invasiveness of conventional laparoscopy [6].

A MEDLINE (Pubmed Central database) search (May 14, 2011) using the keywords 'cholecystectomy' and 'single port', 'single incision', 'single site' or 'single provided 334 articles. However, there were only two randomized controlled studies which were published. These included a low number of patients and focused on postoperative pain $[7,8]$. Current data show that SPLC is feasible with a low rate of conversion to 4-Port conventional laparoscopic cholecystectomy (4PLC) ranging from 2 to $9.3 \%$. The main reason for conversion was the presence of acute inflammation $[9,10]$. Systemic reviews showed an incidence of biliary tract injury between 0.09 and $0.7 \%$. [9-11]. However, high evidence regarding safety of SPLC is missing.

SPLC is regarded to provide excellent results in cosmesis with almost no visible scar $[12,13]$. Yet, the importance of the cosmesis has been challenged as patients also seem to be quite satisfied after 4PLC [14].

Since the available devices for SPLC have been designed to enable an almost scarless procedure the hypothesis of cosmesis improvement has to be proven. No previously published RCT on this topic was designed with the focus on cosmesis. We therefore designed a double-blinded randomized controlled trial on SPLC versus 4PLC (SPOCCtrial) evaluating cosmesis and body image as a primary endpoint.

\section{Methods/Design}

\section{Study objectives}

The SPOCC trial is a prospective, multi-center, double blinded, randomized controlled study comparing SinglePort laparoscopic cholecystectomy with 4-port conventional laparoscopic cholecystectomy in elective surgery for symptomatic cholecystolithiasis. Patients with an indication for an elective cholecystectomy are randomized for either (A) single-port laparoscopic cholecystectomy (SPLC) or (B) conventional 4-port laparoscopic cholecystectomy (4PLC). The hypothesis of this trial is that patients undergoing SPLC will have a better outcome in cosmesis and body image 12 weeks after surgery.

\section{Endpoints}

The primary endpoint of the study concerns patient's satisfaction with cosmesis and body image 12 weeks after surgery. This endpoint is assessed using a validated cosmesis and body image score (CBIS) that was previously used in surgery for Crohn's disease [15] and in donor nephrectomy [16]. This score is calculated on an 8-item multiplechoice type questionnaire (Table 1) ranging between 8 and 48 points.

The secondary endpoint is a combination of several variables: the quality of life 12 weeks after surgery
Table 1 Cosmesis and Body image questionnaire consisting of a body image score (items 1 to 5) and a cosmetic score (items 6 to 8 )

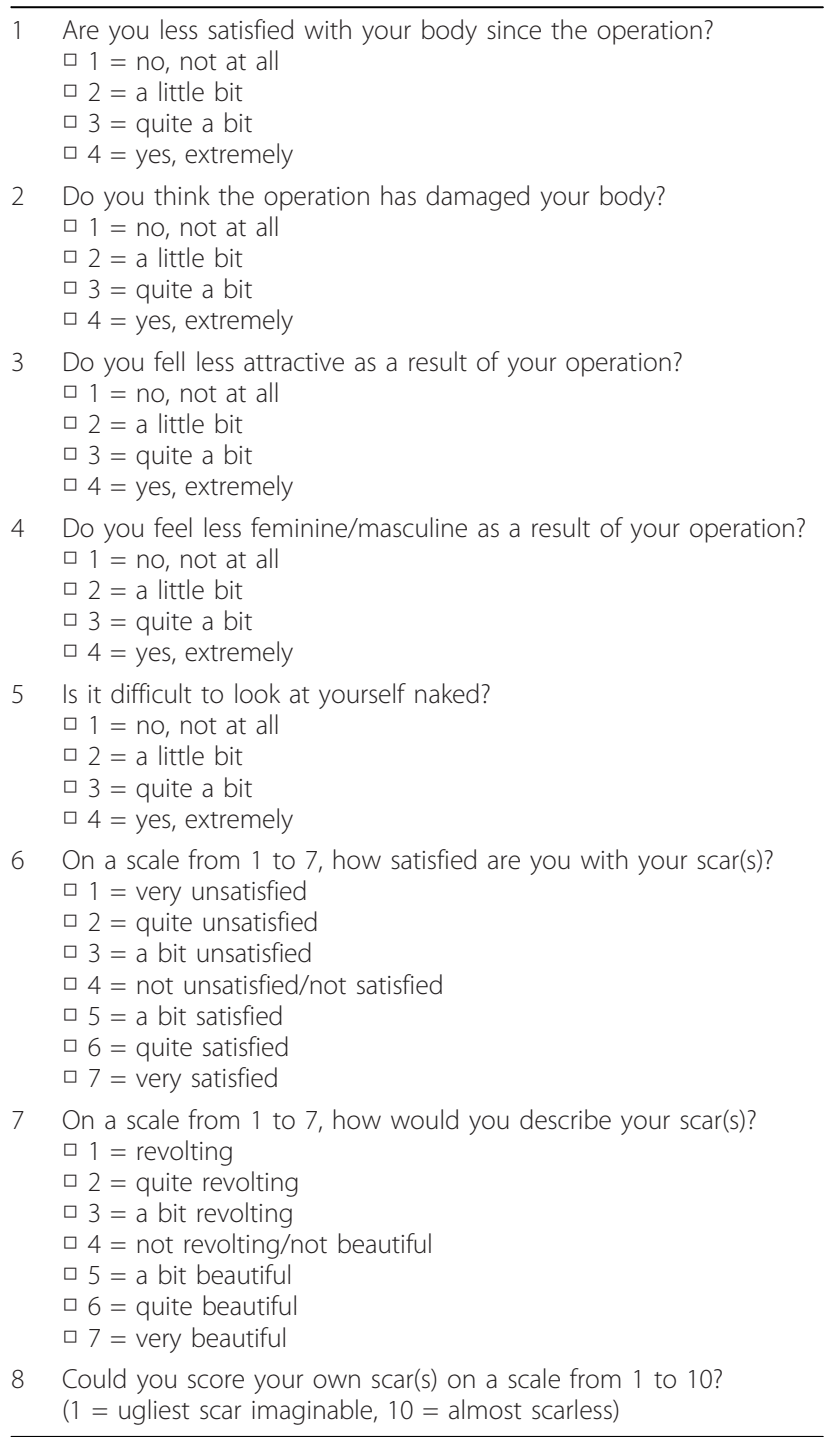

Scores from 8 to 48 .

assessed by the validated Short-Form-36 Health Survey questionnaire [17], postoperative pain assessed by a visual analogue scale daily assessment and the use of analgesics. Operative time, use of additional ports, conversion to open cholecystectomy, length of stay, costs, elapsed time before going back to work as well as intra- and postoperative complications represent aspects of the secondary endpoint. The postoperative complications are classified according to the Clavien-Dindo system [18].

\section{Power of the study}

A clinically relevant improvement of the cosmesis and body image score (CBIS) [15] is defined as an improvement of $20 \%$ of the cosmesis score ( 8 points). Given the reported standard deviation of the CBIS between 4-6 
[19] and using (alpha $=0.05$ and beta $=0.90$ ), two groups of 49 patients are needed. This is based on a two-sided significance level (alpha) of 0.05 and a power of 0.90 . Estimating a $10 \%$ dropout rate, which is common in randomized controlled trials [20], 55 patients will be randomized per arm.

\section{Inclusion criteria}

Inclusion criteria are

- elective cholecystectomy

- 18 years of age

- ability to understand the study purpose (German language)

- written informed consent

\section{Exclusion criteria}

Exclusion criteria are

- pregnant women

- proven or suspected liver cirrhosis

- coagulopathy (platelet count below 50'000/ $\mu \mathrm{l}$ )

- double medication on platelet antagonists (acetylsalicylic acid and clopidogrel)

- international normalized ratio (INR) below 1.4

- Non-German speakers

- patients who are unable to understand the study purpose.

- family members of study investigators or employees of the participating centres

\section{Participating surgeons and clinics}

To prevent surgeon bias, the requirements to qualify as a surgeon are equal in both arms. Since the present study represents a so-called pragmatic randomised control trial [21], the study should reflect daily practice for cholecystectomy. Operating surgeons are senior residents, chief residents, attending surgeons as well as consultant surgeons. The operating surgeon will need a minimal experience of 35 4PLC cholecystectomies, 10 SPLC and must participate in a one-day workshop for SPLC.

Participating centres are the Division of Visceral and Transplantation Surgery of the University Hospital Zurich and the Department of Surgery of the Cantonal Hospital in Winterthur, Switzerland.

\section{Randomization}

Patients have to sign an informed consent in the outpatient clinic. Randomization is performed online (internet randomization module http://www.randomizer.at[22] at the time of transfer to the operating room.

\section{Double blinding}

Patients as well as surgeons, treating physicians and nurses are blinded until the $7^{\text {th }}$ postoperative day. At the end of the operation an opaque wound dressing will be applied at the four port-sites for 4PLC in both treatment arms. The dressings are occlusive and water-resistant. The skin will be closed with subcutaneous absorbable stitches. Additionally the wound will be sealed using fibrin glue to avoid any blood spots on the dressing. Each dressing will be marked with a sterile marker so that any violation of the blinding will be noticed and documented. Data collection as well as clinical controls of patients is performed by investigative independent physicians.

Any change of dressings before 7 days postoperatively are documented.

\section{Data collection and statistics}

Informed consent of patients is performed in the outpatient clinic. Single port cholecystectomy is not offered to patients outside of the study. Data including results of questionnaires is collected on an internet-based secured study information platform [23]. The CBIS questionnaire is filled out at the outpatient clinic preoperatively as well as on the day of admission, 1 and 12 weeks postoperatively. The SF-36 quality of life questionnaire is filled out preoperatively in the outpatient clinic and 12 weeks postoperatively. Pain variables are assessed on a daily basis during hospital stay (Figure 1). Data analysis is performed in accordance with the intention-to-treat principle. Groups are compared using an Independent Samples T-test, Wilcoxon test, or Chi-square test.

\section{Ethics}

This study is conducted in accordance with the principles of the Declaration of Helsinki and 'good clinical practice' guidelines. The principal investigator has completed a course in 'good clinical practice' certified by the Swiss Agency for Therapeutic Products (Swissmedic). The independent medical ethics committee of canton Zurich (Kantonale Ethikkommission Zürich, Switzerland) has approved the study protocol. This trial was registered at clinicaltrial.gov on January 14, 2011 (trial number: NCT01278472).

\section{Surgical technique}

Patients are placed in the French position with the surgeon between the legs, the cameraman (first assistant) on the left of the patient, the second assistant (facultative) on the right, the nurse near the right hand of the surgeon, and the monitor shoulder level to the patient. The endoscopic equipment in terms of optic, monitor, gas supply, suction device, graspers, monopolar hook and endobag are equal in both groups. For surgical disinfection of the skin iodopovidone is used.

\section{Single-Port laparoscopic cholecystectomy (SPLC)}

A transumbilical straight 20 to $25 \mathrm{~mm}$ skin and fascia incision is performed (Figure 2). The peritoneum is 


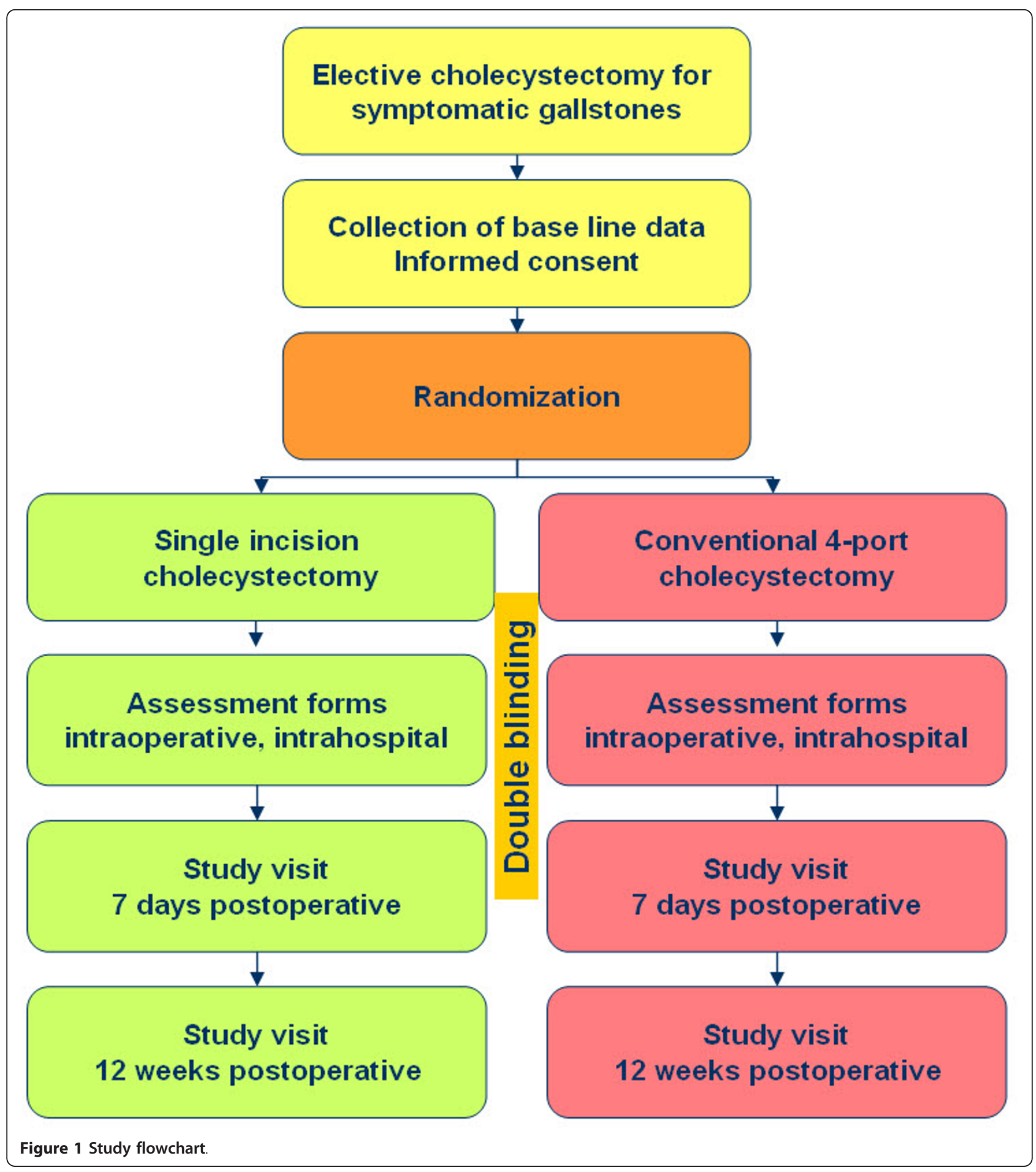

opened and a SILSTM PT12 Port (Covidien Inc., Norwalk, California, USA) is introduced. This port obtains four openings: one for gas insufflation and three that can accommodate trocars ranging from sizes 5 to $12 \mathrm{~mm}$. The compressibility of the elastic polymer allows for the access ports to expand and form fit the space in which it resides as well enabling the ports to pass through the working channels. The pneumoperitoneum is maintained at $10-12 \mathrm{mmHg}$. A $5-\mathrm{mm} 45^{\circ}$ long scope (HD EndoEye ${ }^{\mathrm{TM}}$, Olympus Europa Holding $\mathrm{GmbH}$, Hamburg, Germany) is introduced through one of the openings in the SILSTM Port. The patient is then placed in an anti-Trendelenburg position. The fundus of the gallbladder is grasped and the EndoGrab ${ }^{\mathrm{TM}}$ (Virtual 


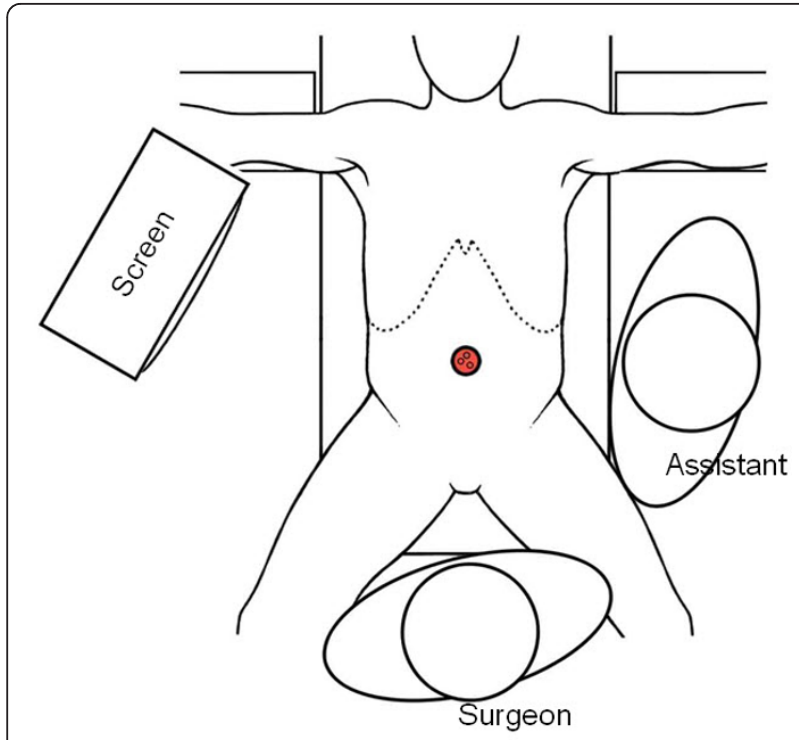

Figure 2 Setting in Single-Port laparoscopic cholecystectomy.

Ports Ltd., Caesarea, Israel) internally anchored retraction system is introduced and placed at the fundus and the peritoneum parietal. The infundibulum is laterally retracted using a bending grasper (Endograsp roticulator ${ }^{\mathrm{TM}}$, Covidien Inc., Norwalk, California, USA). Subsequently good exposure of the triangle of Callot is achieved. The dissection is done using a monopolar dissection hook. The cystic artery and duct are first dissected and then separately clipped with a standard $5 \mathrm{~mm}$ clip applicator (Covidien, Norwalk, California, USA). The gallbladder is pushed upright and dissected free from the liver by means of the monopolar hook. Once the gallbladder is free from the adjacent tissues, the $5 \mathrm{~mm}$ trocar is exchanged for a $10 \mathrm{~mm}$ trocar which is inserted through the SILS ${ }^{\mathrm{TM}}$ access device. An Endocatch bag (Endocatch Gold, 10 mm; Covidien Inc., Norwalk, California, USA) is inserted and the gallbladder is extracted. The umbilical fascia is closed using absorbable Vicryl suture, and the natural scar of the umbilicus is restored using intracutaneous stitches and finally covered by dermal glue (Dermabond ${ }^{\mathrm{TM}}$; Ethicon Endosurgery Inc., Smithfield, RI, USA). The SPLC technique which was used, was presented as a video at the $98^{\text {th }}$ congress of the Swiss Society of Surgery [24].

\section{4-Port laparoscopic cholecystectomy}

A $12 \mathrm{~mm}$ paraumbilical skin incision is performed, the fascia and the peritoneum are incised in an open approach and a $12 \mathrm{~mm}$ Hasson-type blunt trocar (Ethicon Endosurgery Inc., Smithfield, RI, USA) is introduced. The pneumoperitoneum is maintained at $10-12 \mathrm{mmHg}$. A $10 \mathrm{~mm} 45^{\circ}$ long scope (HD EndoEye ${ }^{\mathrm{TM}}$, Olympus Europa Holding GmbH, Hamburg, Germany) is introduced. Now additional $5 \mathrm{~mm}$ trocars are placed under optical control subxyphoidal on the left side of the falciforme ligament and in the right lateral upper abdomen. These trocars are used as endograspers to retain the fundus and the infundibulum of the gallbladder. A further $10 \mathrm{~mm}$ reusable trocar is placed in the left middle abdomen. This trocar is used as dissection instruments, swabs and the endobag (Figure 3). Optimal exposure of the triangle of Callot is achieved. The cystic artery and duct are first dissected and then separately clipped with a standard $5 \mathrm{~mm}$ clip applicator (Covidien, Norwalk, California, USA). The gallbladder is pushed upright and dissected free from the liver by means of the monopolar hook. Once the gallbladder is free from the adjacent tissues a standard Endocatch bag (Endocatch Gold, $10 \mathrm{~mm}$; Covidien, Norwalk, CT, USA) is inserted and the gallbladder is extracted. The paraumbilical fascia und the fascia in the left middle abdomen is closed using absorbable Vicryl suture. The skin is closed using intracutaneous stitches and covered by dermal glue (Dermabond; Ethicon Endosurgery, Inc., Smithfield, RI, USA).

\section{Discussion}

The prevalence of diagnosed cholecystolithiasis is high: $14.4 \%$ in men and $25.3 \%$ in women. About half of these patients are symptomatic and therefore candidates for cholecystectomy [25]. Laparoscopic cholecystectomy has become one of the most frequently performed procedures in visceral surgery. Current efforts focus on minimizing the operative trauma and improving cosmesis by reduction of trocars. On the other hand innovations in this field represent a significant economic burden. Hence, the postulated advantages for the patients of SPLC as well as cost effectiveness need to be evaluated in prospective randomized studies.

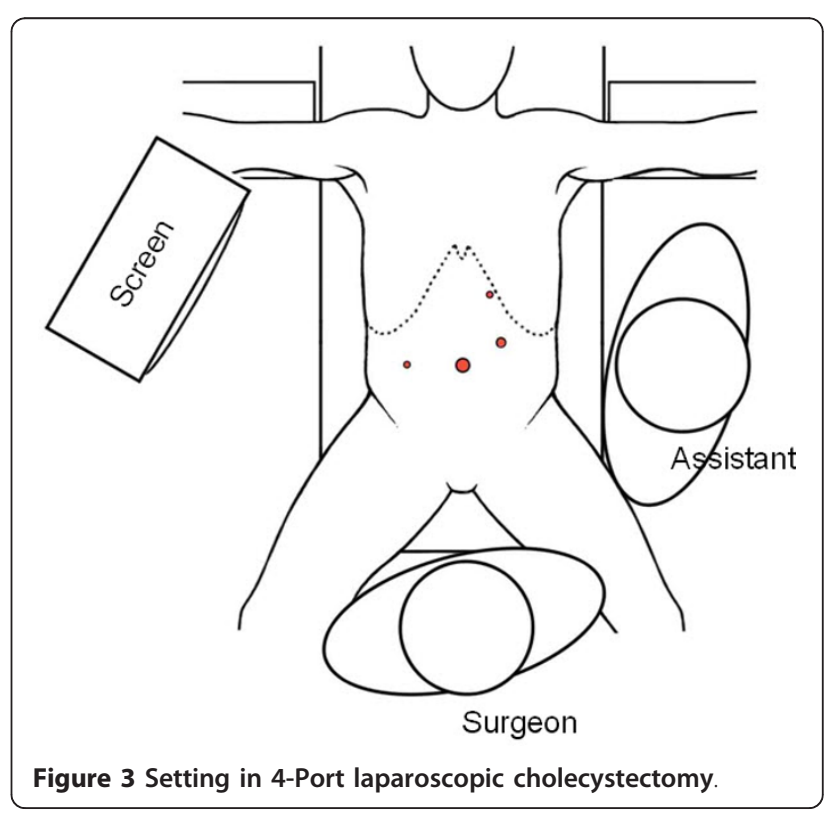


Table 2 Listed recruiting Randomized Controlled Trials (RCT) on clinicaltrial.gov (search date: May-14, 2011). QoL = Quality of life

\begin{tabular}{|c|c|c|c|c|c|}
\hline trial number & study Center & enrollment & $1^{\circ}$ endpoint & $2^{\circ}$ endpoint(s) & masking \\
\hline NCT01348620 & Sacro Cuore Don Calabria & 58 & Pain & Cosmesis (VAS) & Double blind \\
\hline NCT01094379 & Athens & 40 & Pain & Cosmesis ( 1 month), OR time, QoL & Single blind \\
\hline NCT01104727 & EAES & 600 & Morbidity rate & Pain, cosmesis (2 months) & Open label \\
\hline NCT00974194 & Lausanne & 260 & Pain & $\begin{array}{l}\text { Complications, costs, cosmesis }(1,3 \text {, } \\
12 \text { months), complications }\end{array}$ & Double blind \\
\hline NCT01268748 & Copenhagen & 120 & Pain & $\begin{array}{l}\text { Cosmesis ( } 1 \text { month), well-being } \\
\text { score }\end{array}$ & Double blind \\
\hline NCT01195285 & Saint Luke's Health System & 150 & Costs & Pain, morbidity, QoL & Double blind \\
\hline NCT00904865 & Geneva & 200 & $\begin{array}{l}\text { Cosmesis, body } \\
\text { image and QoL } \\
\text { (1 month) }\end{array}$ & Morbidity, Pain, OR time & Open label \\
\hline NCT01339325 & San Giovanni Addolorata & 180 & $\begin{array}{l}\text { Compound of length } \\
\text { of stay, pain, cosmesis } \\
\text { and QoL }\end{array}$ & $\begin{array}{l}\text { OR time, conversion rate, } \\
\text { complication rate }\end{array}$ & Open label \\
\hline NCT01278472 & Zurich & 110 & Cosmesis (3 months) & $\begin{array}{l}\text { Pain, QoL, conversion rate, } \\
\text { convalescence }\end{array}$ & Double blind \\
\hline
\end{tabular}

While many retrospective case series on SPLC are available $[9,10]$, only two RCTs have been published showing reduced postoperative pain $[8,26]$.

Currently 9 RCTs are registered in the international trial database (clinicaltrials.gov) (Table 2). Two trials have small sample sizes and focus on postoperative pain (NCT01348620, NCT01094379). The majority of trials focus on postoperative pain or costs. One trial with the most extensive sample size (600-patients) investigates differences in morbidity (Trial of the European Association of Endoscopic Surgery, EAES, NCT01104727).

Cosmesis and body image (after 1 month) as a primary endpoint is considered in only one registered trial (NCT00904865) while another study focuses on a combined primary endpoint consisting of quality of life assessed by SF-36, cosmesis and pain (NCT01339325trial) (Table 2).

The present SPOCC-trial has two specific advantages, such as the time point of cosmesis assessment 3 months after surgery, which represents a reasonable follow-up time point, using a validated questionnaire and scoring system [15]. The second strength of the trial design is the double blinding.

\section{Acknowledgements}

Approved by the Medical Ethics Commission of the Canton Zurich, Switzerland, (Kantonale Ethikkommission Zurich). The study is not externally funded by government or charity foundations. No funding or assistance is received from commercial organizations.

\section{Author details}

${ }^{1}$ Department of Surgery, Division of Visceral and Transplantation Surgery, University Hospital Zurich, 8091 Zurich, Switzerland. 'Department of Surgery, Cantonal Hospital Winterthur, 8401 Winterthur, Switzerland.

\section{Authors' contributions}

DCS and SB drafted the manuscript. DCS, GL, CEO and SB designed the protocol and co-authored the writing of the manuscript. DAR contributed to the design of the protocol and calculation of the power of the study. All other authors participated in the design of the study and are local investigators in the participating centers. All authors were involved in editing the manuscript and approved the final text of the manuscript.

\section{Competing interests}

The authors declare that they have no competing interests.

Received: 31 May 2011 Accepted: 12 September 2011

Published: 12 September 2011

\section{References}

1. Litynski GS: Erich Muhe and the rejection of laparoscopic cholecystectomy (1985): a surgeon ahead of his time. JSLS 1998, 2(4):341-346.

2. Begos DG, Modlin IM: Laparoscopic cholecystectomy: from gimmick to gold standard. J Clin Gastroenterol 1994, 19(4):325-330.

3. Scott DJ, Tang SJ, Fernandez R, Bergs R, Goova MT, Zeltser I, Kehdy FJ, Cadeddu JA: Completely transvaginal NOTES cholecystectomy using magnetically anchored instruments. Surg Endosc 2007, 21(12):2308-2316.

4. Pearl JP, Ponsky JL: Natural orifice translumenal endoscopic surgery: a critical review. J Gastrointest Surg 2008, 12(7):1293-1300.

5. Rao PP, Bhagwat $S M$, Rane A: The feasibility of single port laparoscopic cholecystectomy: a pilot study of 20 cases. HPB (Oxford) 2008, 10(5):336-340.

6. Schlager A, Khalaileh A, Shussman N, Elazary R, Keidar A, Pikarsky AJ, BenShushan A, Shibolet O, Horgan S, Talamini M, Zamir G, Rivkind Al, Mintz Y: Providing more through less: current methods of retraction in SIMIS and NOTES cholecystectomy. Surg Endosc 2010, 24(7):1542-1546.

7. Ma J, Cassera MA, Spaun GO, Hammill CW, Hansen PD, Aliabadi-Wahle S: Randomized Controlled Trial Comparing Single-Port Laparoscopic Cholecystectomy and 4-Port Laparoscopic Cholecystectomy. Ann Surg 2011.

8. Tsimoyiannis EC, Tsimogiannis KE, Pappas-Gogos G, Farantos C, Benetatos N, Mavridou P, Manataki A: Different pain scores in single transumbilical incision laparoscopic cholecystectomy versus classic laparoscopic cholecystectomy: a randomized controlled trial. Surg Endosc 24(8):1842-1848.

9. Allemann $P$, Schafer M, Demartines N: Critical appraisal of single port access cholecystectomy. Br J Surg 2010, 97(10):1476-1480. 
10. Antoniou SA, Pointner R, Granderath FA: Single-incision laparoscopic cholecystectomy: a systematic review. Surg Endosc 2011, 25(2):367-377.

11. Giger U, Ouaissi M, Schmitz SF, Krahenbuhl S, Krahenbuhl L: Bile duct injury and use of cholangiography during laparoscopic cholecystectomy. Br J Surg 2011, 98(3):391-396.

12. Tacchino R, Greco F, Matera D: Single-incision laparoscopic cholecystectomy: surgery without a visible scar. Surg Endosc 2009, 23(4):896-899.

13. Chow A, Purkayastha S, Aziz O, Paraskeva P: Single-incision laparoscopic surgery for cholecystectomy: an evolving technique. Surg Endosc 2010, 24(3):709-714.

14. Bignell M, Hindmarsh A, Nageswaran H, Mothe B, Jenkinson A, Mahon D, Rhodes M: Assessment of cosmetic outcome after laparoscopic cholecystectomy among women 4 years after laparoscopic cholecystectomy: is there a problem? Surg Endosc 2011

15. Dunker MS, Stiggelbout AM, van Hogezand RA, Ringers J, Griffioen G, Bemelman WA: Cosmesis and body image after laparoscopic-assisted and open ileocolic resection for Crohn's disease. Surg Endosc 1998, 12(11):1334-1340.

16. Lind MY, Hop WC, Weimar W, JN IJ: Body image after laparoscopic or open donor nephrectomy. Surg Endosc 2004, 18(8):1276-1279.

17. Ware JE, Sherbourne CD: The MOS 36-item short-form health survey (SF36). I. Conceptual framework and item selection. Med Care 1992, 30(6):473-483.

18. Dindo D, Demartines $N$, Clavien PA: Classification of surgical complications: a new proposal with evaluation in a cohort of 6336 patients and results of a survey. Ann Surg 2004, 240(2):205-213.

19. Keus F, de Vries J, Gooszen HG, van Laarhoven CJ: Laparoscopic versus small-incision cholecystectomy: health status in a blind randomised trial. Surg Endosc 2008, 22(7):1649-1659.

20. Lurie I, Levine SZ: Meta-analysis of dropout rates in SSRIs versus placebo in randomized clinical trials of PTSD. J Nerv Ment Dis 198(2):116-124.

21. Ergina PL, Cook JA, Blazeby JM, Boutron I, Clavien PA, Reeves BC, Seiler CM, Altman DG, Aronson JK, Barkun JS, Campbell WB, Feldman LS, Flum DR, Glasziou P, Maddern GJ, Marshall JC, McCulloch P, Nicholl J, Strasberg SM, Meakins JL, Ashby D, Black N, Bunker J, Burton M, Campbell M, Chalkidou K, Chalmers I, de Leval M, Deeks J, Grant A, et al: Challenges in evaluating surgical innovation. Lancet 2009, 374(9695):1097-1104.

22. [http://www.randomizer.at]

23. [http://www.single-port-rct.com/].

24. Steinemann DC, Clavien PA, Breitenstein S: Single-Port cholecystectomy using internal retraction. swissknife 2011, s1.

25. Volzke H, Robinson DM, John U: Association between thyroid function and gallstone disease. World J Gastroenterol 2005, 11(35):5530-5534.

26. Asakuma M, Hayashi M, Komeda K, Shimizu T, Hirokawa F, Miyamoto Y, Okuda J, Tanigawa N: Impact of single-port cholecystectomy on postoperative pain. Br J Surg 2011, 98(7):991-995.

\section{Pre-publication history}

The pre-publication history for this paper can be accessed here: http://www.biomedcentral.com/1471-2482/11/24/prepub

doi:10.1186/1471-2482-11-24

Cite this article as: Steinemann et al:: Cosmesis and body image after single-port laparoscopic or conventional laparoscopic cholecystectomy: a multicenter double blinded randomised controlled trial (SPOCC-trial). BMC Surgery 2011 11:24.

\section{Submit your next manuscript to BioMed Central and take full advantage of:}

- Convenient online submission

- Thorough peer review

- No space constraints or color figure charges

- Immediate publication on acceptance

- Inclusion in PubMed, CAS, Scopus and Google Scholar

- Research which is freely available for redistribution 